\title{
A possible interface between autonomic function and pain control: opioid analgesia and the nucleus tractus solitarius
}

\author{
J.W. Lewis, G. Baldrighi and H. Akil \\ Mental Health Research Institute, Department of Psychiatry, University of Michigan, Ann Arbor, MI 48109 (U.S.A.)
}

(Accepted 24 March 1987)

Key words: Analgesia; Stimulation-produced analgesia; Opioid peptide; Naloxone; Nucleus tractus solitarius; Pain; Pain-inhibition

\begin{abstract}
Opioid peptides appear to be important neurochemical mediators in central nervous system mechanisms of analgesia, cardiovascular control, and many endocrinological responses to stress. The nucleus tractus solitarius (NTS), a brain region expressing all 3 opioid peptide families, is also associated with regulation of autonomic and endocrine functions. We now report that electrical stimulation of the NTS causes pronounced analgesia in rats. This analgesia appears to involve opioids and is pharmacologically dissociable from the hemodynamic changes elicited by NTS stimulation. These results suggest the NTS as a neural substrate for inter-relationships between stress, cardiovascular function, alterations in respiration, and pain sensitivity.
\end{abstract}

\section{INTRODUCTION}

Intimate associations between pain sensitivity, cardiovascular function, opioid peptides, and the response to stress are now beginning to be revealed ${ }^{10}$, ${ }^{28}$. The nucleus tractus solitarius (NTS), located in the caudal medulla and well-known to be involved in cardiovascular and respiratory regulation (e.g. ref. 8) may be a neural locus for these interactions. Converging lines of evidence have led us to hypothesize that the NTS could also be an integral part of endogenous pain-inhibitory systems that involve opioid peptides. Biochemically, this region contains opioid receptors and peptides derived from all 3 opioid precursor families: pro-opiomelanocortin, pro-enkephalin, and pro-dynorphin ${ }^{2,11,12,18,32}$. Anatomically, the NTS has extensive projections to, or receives afferents from, several brain loci thought to be involved in pain and pain-inhibition (e.g., periaqueductal/periventricular gray matter, nucleus raphe magnus, and the spinal cord) ${ }^{23,29}$. Behaviorally, microinjection of morphine ${ }^{24}$ or noradrenergic agents ${ }^{21}$ into the NTS causes analgesia.
We now report that electrical stimulation of the NTS causes opioid-mediated analgesia in pentobarbital-anesthetized rats. Thus, while the NTS is not classically thought of as being along the pain pathway, our findings suggest the existence of a close structural and functional interface between cardiovascular/respiratory control and regulation of responsiveness to noxious stimuli. This interface appears to take place at a very early autonomic relay station in the central nervous system. A preliminary report of these data has been made ${ }^{13}$.

\section{MATERIALS AND METHODS}

\section{Experiment 1. Analgesic effect of NTS stimulation}

Subjects were male Sprague-Dawley rats (200-250 g; Charles River Laboratories, Worcester, MA). Pain sensitivity was assessed using the tail-flick test ${ }^{7}$ with a maximum response latency of $10 \mathrm{~s}$ imposed to minimize tissue damage. In the present experiments, the analgesic effect of brain stimulation was determined in rats anesthetized with sodium pentobarbital. That behavioral measurements ob- 
tained in anesthetized animals reflect responses in the awake state is supported by the fact that tail-flick latencies in the anesthetized rats are similar, or slightly decreased, compared to those exhibited by awake rats and by findings that stimuli which modify tail-flick latencies (e.g. electrical brain stimulation, footshock, morphine) cause similar behavioral

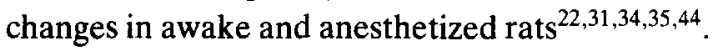

Rats were anesthetized with sodium pentobarbital $(50 \mathrm{mg} / \mathrm{kg}$, i.p.) and mounted in a stereotaxic frame. The surface of the brainstem at the level of the obex was exposed and the stimulating electrode (a twisted pair of nichrome enamel insulated wires $0.125 \mathrm{~mm}$ diameter) lowered approximately $1.0-1.5 \mathrm{~mm}$ into the brain. The electrical stimulus was produced using a Grass model S88 stimulator and consisted of monopolar, biphasic square-wave pulses ( $50 \mu$ s pulses separated by a $100-\mu$ s interval) delivered at a rate of 20 pulses/s and ranging in intensity from 125 to $750 \mu \mathrm{A}$. (Note: caution must be exercised in direct comparison of brain stimulation threshold values obtained by various researchers. While these intensities of stimulation used herein may appear high, the pulse duration is very brief.)

Prior to brain stimulation, baseline tail-flick latencies were determined at 1-min intervals until a stable response was obtained. Electrical stimulation, beginning with an intensity of $125 \mu \mathrm{A}$ was then delivered for $30 \mathrm{~s}$. Tail-flick testing resumed immediately upon termination of the stimulation and continued with trials at 1-min intervals until tail-flick latencies returned to baseline levels. If analgesia was not observed (i.e. poststimulation response latency was less than 1.5 times the baseline latency), the stimulation current was increased by $125 \mu \mathrm{A}$, and this procedure was repeated. If no analgesia was obtained at the maximal stimulation intensity of $750 \mu \mathrm{A}$, the electrode was repositioned and testing begun again. At some electrode placements excessive motor stimulation (e.g. twitching of the limbs, arching of the back) or cessation of respiration were observed; when these changes occurred the electrode was repositioned. During the behavioral testing procedures, animals were given additional anesthetic $(10.0 \mathrm{mg} / \mathrm{kg}$, i.p. $)$ as needed.

When stimulation at a particular site was found to cause an increase in tail-flick latency, pharmacological characterization to this analgesia was begun. Ani- mals were treated according to one of two protocols. After determination on the threshold current for eliciting analgesia, some rats $(n=6)$ were injected with physiological saline $(1 \mathrm{ml} / \mathrm{kg}, \mathrm{s.c}$.). After $10 \mathrm{~min}$, baseline tail-flick latencies were determined and the threshold stimulation current previously found to elicit analgesia was applied. If this stimulus still caused elevations in tail-flick latencies, the animals were then injected with the opiate antagonist, naloxone $(10 \mathrm{mg} / \mathrm{kg}, \mathrm{s.c}$ ), and the testing procedure repeated $10 \mathrm{~min}$ later. To control for factors that may have affected the behavior of this group of rats, such as duration of anesthesia or possible effects of repeated brain stimulation, a second group of rats $(n=$ 6) was tested in a similar manner but without the saline injection.

To determine the anatomical specificity of the sites supporting stimulation-produced analgesia, several electrode penetrations (2-4/rat) were made in the region of the NTS in control rats $(n=6)$. In these animals a maximal stimulus $(750 \mu \mathrm{A})$ was applied and alterations in tail-flick latencies noted. Although the purpose of the group was to demonstrate that stimulation of structures adjacent to the NTS does not cause analgesia, a stimulation site that did cause increases in tail-flick latencies was located in each rat, attesting to the viability of each preparation.

A the end of each experimental session, the stimulation site(s) was marked with an electrolytic lesion, the animals perfused intracardially with a saline/formalin solution, and the brain prepared with Nissl stains for histological verification of the electrode site. Location of the electrode was evaluated using the atlas of Paxinos and Watson ${ }^{26}$. Behavioral data were analyzed with Student's $t$-test for dependent samples.

\section{Experiment 2. Relationship between NTS stimulation- produced analgesia and hemodynamic changes}

To assess the possible relationship between NTS stimulation-produced analgesia and hemodynamic changes, we examined simultaneously the effects of NTS stimulation on pain sensitivity and arterial blood pressure. Rats $(n=8)$ were prepared for brain stimulation as before and the femoral artery catheterized for measurement of blood pressure. Blood pressure was recorded using a Statham Laboratories pressure transducer (Model P23AC) and a Grass polygraph. 
Brain stimulation and behavioral testing procedures were carried out as before.

Observations from each stimulation site were classified non-parametrically on two dimensions: analgesia vs no analgesia, and blood pressure increased, decreased, or did not change. To test for a possible association between blood pressure change and analgesia, these data were analyzed statistically using a Chisquare test.

Finally, in some of the rats catheterized for blood pressure measurements, further dissociation of the analgesic and hemodynamic effects of NTS stimulation was accomplished pharmacologically. In each rat, a stimulation site was located that elicited increases in tail-flick latency accompanied by hypertension (the most common effect of NTS stimulation). Rats $(n=3)$ were then given either trimethaphan camsylate (Arfonad; Roche Laboratories, Nutley $\mathrm{NJ} ; 4 \mathrm{mg} / \mathrm{kg}$, i.v.), a peripherally acting antagonist of sympathetic nervous system activity, or naloxone $(10 \mathrm{mg} / \mathrm{kg} ; n=3)$. Testing resumed $10-20 \mathrm{~min}$ later and the effects on blood pressure and pain sensitivity of threshold stimulation observed.

\section{RESULTS}

Stimulation of those sites in or near NTS (Fig. 1 top, $n=12$ ) was found to elicit marked elevations in tail-flick latencies, whereas stimulation of placements dorsal, lateral, or ventral to this nucleus (Fig. 1 top, $n=12$ ) was without effect. Following threshold stimulation of NTS sites, tail-flick latencies were significantly elevated compared to baseline values $(P<$ 0.01 ; Fig. 1 bottom, A,B). The duration of stimulation-produced analgesia was variable across subjects. Defining analgesia as an increase in tail-flick latency of at least 1.5 times the baseline value, poststimulation analgesia lasted between 1 and 9 min. Neither baseline nor poststimulation latencies were significantly altered following saline injections (Fig. 1 bottom, B). Naloxone administration, by contrast, significantly attenuated the analgesic response to threshold stimulation $(P<0.01$, compared to either postsaline or threshold latencies; Fig. 1 bottom, A,B).

By varying stimulation site and intensity, a total of 167 measurements of poststimulation blood pressure and pain sensitivity were obtained from the 8 rats

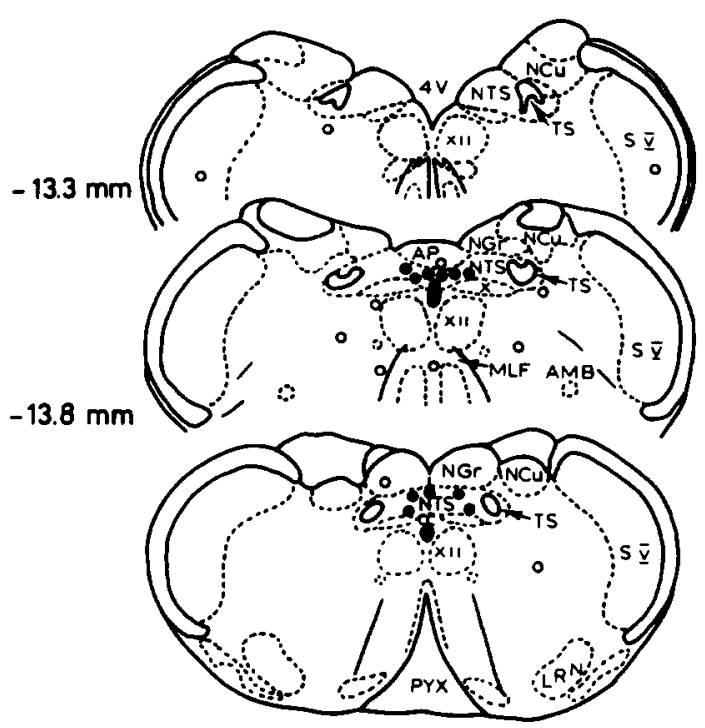

Bregma $-14.3 \mathrm{~mm}$
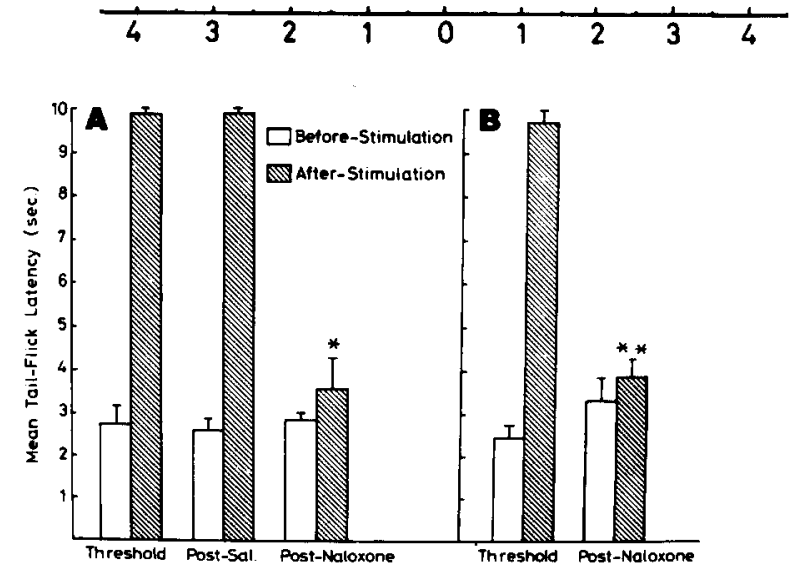

Fig. 1. Top: location of stimulation sites. Sites that supported analgesia are depicted by closed circles $(n=12)$ and ineffective sites are indicated by open circles $(n=12)$. Bottom: effect of NTS stimulation on tail-flick latency. Panels A and B show data from rats tested for pain sensitivity before and after NTS stimulation. NTS stimulation markedly increased tail-flick latency compared to baseline values (A and B). Injection of saline did not affect NTS stimulation-produced analgesia (B). Naloxone, markedly attenuated stimulation-produced analgesia $\left(\mathrm{A},{ }^{*}\right.$ differed from both postthreshold and postsaline latencies, $P<$ $0.01 ; \mathrm{B},{ }^{* *}$ differed from postthreshold latency, $P<0.01$ ). AMB, ambiguus nucleus; $4 \mathrm{~V}$, fourth ventricle; LRN, lateral reticular nucleus; NTS, nucleus tractus solitarius; NCu, nucleus cuneatus; PYX, decussation of the pyramidal tract; S V, spinal trigeminal nucleus; TS, tractus solitarius; $X$, dorsal motor nucleus of vagus; XII, hypoglossal nucleus.

tested. These data are presented in Table I. Statistical analysis indicated no significant association between changes in blood pressure and analgesia ( $\mathrm{Chi}$ square $=1.38, P>0.05$ ). Furthermore, blockade of 


\section{TABLE I}

Classification of the effects of electrical stimulation in and around the NTS on arterial blood pressure and pain sensitivity

These data summarize the effects of 167 stimulation sites. There was no significant association between elicitation of analgesia and alterations in blood pressure $($ Chi square $=1.38$, $P>0.05)$

\begin{tabular}{llll}
\hline & \multicolumn{3}{l}{ Arterial blood pressure } \\
\cline { 2 - 4 } & Decrease & No change & Increase \\
\hline Analgesia & 14 & 11 & 45 \\
No analgesia & 26 & 17 & 54 \\
\hline
\end{tabular}

sympathetic activity with Arfonad eliminated the pressor response without affecting analgesia. At similar stimulation sites in other rats, treatment with naloxone blocked stimulation-produced analgesia without markedly affecting the hypertension.

\section{DISCUSSION}

Taken together, these findings demonstrate that electrical stimulation of the nucleus tractus solitarius causes analgesia that is prevented by naloxone suggesting mediation by opioid peptides. That control stimulation sites, near the NTS, were ineffective in causing analgesia indicates that the present results are not due to stimulation of adjacent structures, many of which have been implicated in mechanisms of pain-inhibition (e.g. the dorsolateral funiculus ${ }^{4}$ or the dorsal columns ${ }^{33}$ ). Comparison of stimulationproduced analgesia elicited from the NTS to those forms previously reported following stimulation of more classical pain-inhibitory loci $\mathrm{i}^{3,6,20,25}$ suggests that NTS-derived analgesia is comparable in magnitude with these other forms of brain stimulation-produced analgesia, but that it is typically of longer duration and is more sensitive to antagonism by naloxone. Perhaps the latter finding indicates the production of a more 'pure' opioid analgesia.

That the pain regulatory and cardiovascular mechanisms can be dissociated pharmacologically or by manipulating the stimulation site, provides evidence that these responses are subserved by separable neural elements and that increases in tail-flick latency are not secondary to alterations in blood pressure, per se. This dissociation between analgesia and cardiovascular changes is congruent with previous re- ports indicating the independence of opioid-mediated decreases in pain sensitivity and hyperten$\operatorname{sion}^{30,42,43}$. These results, however, do not imply that antinociception and blood pressure are independent events in response to natural stimuli. Moreover, since analgesia has been associated with increases and decreases in blood pressure, it may be that the critical signal for stimulation of opioid analgesia systems is deviation from homeostasis. It should be noted that another effect of NTS stimulation, alteration in respiration, may be linked to changes in painsensitivity. The present study, however, did not examine this variable systematically. Nevertheless, the fact that blood pressure control, respiratory regulation, and antinociception share common anatomical, and possibly biochemical, substrates may begin to reveal a neural basis for reported changes in pain sensitivity accompanying stress, cardiovascular hypertension $^{8,10,28}$, and decreased pain-responsiveness induced by alterations in respiratory pattern, such as LaMaze analgesia ${ }^{40}$.

Current conceptions of the organization of an endogenous opioid analgesia system could be significantly affected by integration of the NTS. In general, this analgesia system has been described as arising in the periventricular/periaqueductal gray of the medial brainstem, involving monoaminergic neurons of the nucleus raphe magnus and the nucleus reticularis paragigantocellularis, descending to the spinal cord via the dorsolateral funiculus, and exerting pain-inhibition in the spinal dorsal horn (for reviews see refs. $3,15,39$ ). While this well-defined opioid analgesia system can be activated by direct electrical stimulation or administration of opiate drugs $s^{3,15,20 \text {, }}$ $25,36,37,39,41$, the origin of the neural signals capable of energizing this system in response to environmental stimulation is not known. The NTS is a major recipient of somato/visceral sensory information and anatomically connected to pain/inhibitory regions making it a likely candidate for such a role.

Finally, it may be that the NTS, by providing a direct input to endogenous analgesia systems, serves as a crucial interface between environmental stressors and neural substrates of pain inhibition. For example, in man and animals, exposure to stress appears to be an adequate stimulus for activation of endogenous opioid pain-inhibitory systems (e.g. refs. 1, 5, $16,38)$. One form of opioid-mediated, stress-induced 
analgesia $^{1,14}$ can be attenuated by unilateral vagotomy $^{19}$ or adrenal demedullation ${ }^{17}$, indicating the importance of peripheral autonomic systems in this response. Thus, since exposure to stress causes analgesia and is accompanied by numerous autonomic sequelae, and because the NTS is known to be involved in these autonomic responses and NTS stimulation elicits pain-inhibition, it is reasonable to hypothesize that an important linkage between stressful stimuli and endogenous analgesia systems occurs via the nu-

\section{REFERENCES}

1 Akil, H., Madden, J. III, Patrick, R.L. and Barchas, J.D., Stress-induced increase in endogenous opiate peptides; concurrent analgesia and its partial reversal by naloxone. In H.W. Kosterlitz (Ed.), Opiates and Endogenous Opioid Peptides, Elsevier, Amsterdam, 1976, pp. 63-70.

2 Atweh, S.F. and Kuhar, M.J., Autoradiographic localization of opiate receptors in rat brain. I. Spinal cord and lower medulla, Brain Research, 129 (1977) 53-67.

3 Basbaum, A.I. and Fields, H.L., Endogenous pain control systems: brainstem spinal pathways and endorphin circuitry, Annu. Rev. Neurosci., 7 (1984) 309-338.

4 Basbaum, A.I., Marley, N.J., O'Keefe, J. and Clanton, C.H., Reversal of morphine and stimulus produced analgesia by subtotal spinal cord lesions, Pain, 3 (1977) 43-56.

5 Bodnar, R.J., Types of stress which induce analgesia. In M.D. Tricklebank and G. Curzon (Eds.), Stress Induced Analgesia, Wiley, London, 1984, pp. 19-32.

6 Cannon, J.T., Prieto, G.J., Lee, A. and Liebeskind, J.C., Evidence for opioid and non-opioid forms of stimulationproduced analgesia in the rat, Brain Research, 243 (1982) 315-321.

7 D'Amour, F.E. and Smith, D.L., A method for determining loss of pain sensation, J. Pharmacol. Exp. Ther., 72 (1941) 74-79.

8 Galosy, R.A., Clarke, L.K., Vasco, M.R. and Crawford, I.L., Neurophysiology and neuropharmacology of cardiovascular regulation and stress, Neurosci. Biobehav. Rev., 5 (1981) 137-175.

9 Hassen, A.H., Feurstein, G. and Faden, A.J., Differential cardiovascular effects mediated by mu and kappa opiate receptors in hindbrain nuclei, Peptides, 4 (1983) 621-625.

10 Holaday, J.W., Cardiovascular effects of endogenous opiate systems, Annu. Rev. Pharmacol. Toxicol., 23 (1983) 541-594.

11 Joseph, S.A., Pilcher, W.H. and Bennett-Clarke, C., Immunocytochemical localization of ACTH perikarya in nucleus tractus solitarius: evidence for a second opiocortin neuronal system, Neurosci. Lett., 38 (1983) 221-225.

12 Khachaturian, H., Lewis, M.E., Shafer, M.K.-H. and Watson, S.J., Anatomy of CNS opioid systems, Trends Neurosci., 8 (1985) 111-119.

13 Lewis, J.W., Baldrighi, G., Watson, S.J. and Akil, H., Electrical stimulation of the nucleus tractus solitarius (NTS) causes opioid mediated analgesia in the rat, Soc. Neurosci. Abstr., 11 (1985) 637.

14 Lewis, J.W., Cannon, J.T. and Liebeskind, J.C., Opioid cleus tractus solitarius.

\section{ACKNOWLEDGEMENTS}

We thank Drs. T.G. Sherman, M.E. Lewis, H. Khachaturian, M. Schaffer and S.J. Watson for their helpful comments and Endo Dupont (Wilmington, DE) for the gift of naloxone. Supported by NIDA grant DA02265. J.W.L. was a NIDA postdoctoral fellow, Grant F32 DA05221.

and nonopioid mechanisms of stress analgesia, Science, 208 (1980) 623-625.

15 Lewis, J.W., Khachaturian, H., Lewis, M.E. and Akil, H., Opioid peptides: endogenous analgesics? In J.F. Mitchell, W. Paton and P. Turner (Eds.), Proceedings of the IX International Congress of Pharmacology, MacMillan, London, 1985, pp.

16 Lewis, J.W., Terman, G.W., Nelson, L.R. and Liebeskind, J.C., Opioid and non-opioid stress analgesia. In M.D. Tricklebank and G. Curzon (Eds.), Stress Induced Analgesia, Wiley, London, 1984, pp. 103-134.

17 Lewis, J.W., Tordoff, M.G., Sherman, J.E. and Liebeskind, J.C., Adrenal medullary enkephalin-like peptides may mediate opioid stress analgesia, Science, 217 (1982) 557-559.

18 Lewis, M.E., Khachaturian, H. and Watson, S.J., Combined autoradiographic immunocytochemical analysis of opioid receptors and opioid peptide neuronal systems in the brain, Peptides, 6 (Suppl. 1) (1985) 37-47.

19 Maixner, $W$. and Randich, A., Role of the right vagal nerve trunk in antinociception, Brain Research, 298 (1984) 374-377.

20 Mayer, D.J., Wolfle, T.L., Akil, H., Carder, B. and Liebeskind, J.C., Analgesia from electrical stimulation in the brainstem of the rat, Science, 174 (1971) 1351-1354.

21 Menescal de Oliveira, L. and Lico, M.C., Pain modulation in the adrenergically stimulated area postrema in the alert guinea pig, Physiol. Behav., 19 (1977) 359-364.

22 Morgan, M.M., Depaulis, A. and Liebeskind, J.C., Periaqueductal gray matter stimulation produced analgesia in the pentobarbital anesthetized rat, Proc. West. Pharmacol. Soc., 28 (1985) 161-163.

23 Norgren, R., Projections from the nucleus of the solitary tract in the rat, Neuroscience, 3 (1978) 207-218.

24 Oley, N., Cordova, C., Kelly, M.L. and Bronzino, J.D., Morphine administration to the region of the solitary tract produces analgesia in rats, Brain Research, 236 (1982) 511-516.

25 Oliveras, J.L., Hosobuchi, Y., Redjemi, F., Guilbaud, G. and Besson, J.M., Opiate antagonist, naloxone, strongly reduces analgesia by stimulation of a raphe nucleus (centralis inferior), Brain Research, 120 (1977) 221-229.

26 Paxinos, G. and Watson, C., The Rat Brain in Stereotaxic Coordinates, Academic Press, Sydney, 1982.

27 Petty, M.A. and De Jong, W., Enkephalins induce a centrally mediated rise in blood pressure in rats, Brain Research, 260 (1983) 322-325.

28 Randich, A. and Maixner, W., Interactions between car- 
diovascular and pain regulatory systems, Neurosci. Biobehav. Rev., 8 (1984) 343-367.

29 Ricardo, J.A. and Koh, E.T., Anatomical evidence of direct projections from the nucleus of the solitary tract to the hypothalamus, amygdala, and other forebrain structures in the rat, Brain Research, 153 (1978) 1-26.

30 Saavedra, J.M., Naloxone reversible decrease in pain sensitivity in young and adult spontaneously hypertensive rats, Brain Research, 209 (1981) 245-249.

31 Sandkuhler, J. and Gebhart, G.F., Characterization of inhibition of a spinal nociceptive reflex by stimulation medially and laterally in the midbrain and medulla in the pentobarbital-anesthetized rat, Brain Research, 305 (1984) 67-76.

32 Schwartzberg, D.S. and Nakane, P.K., ACTH-related peptide containing neurons within the medulla oblongata of the rat, Brain Research, 276 (1983) 351-356.

33 Shealy, C.N., Mortimer, J.T. and Hagfors, N.R., Dorsal column electroanalgesia, J. Neurosurg., 32 (1970) $560-564$.

34 Terman, G.W., Pechnick, R. and Liebeskind, J.C., Blockade of tolerance to morphine analgesia by pentobarbital, Proc. West. Pharmacol. Soc., 28 (1985) 157-160.

35 Terman, G.W., Shavit, Y., Lewis, J.W., Cannon, J.T. and Liebeskind, J.C., Intrinsic mechanisms of pain inhibition: activation by stress, Science, 226 (1984) 1270-1277.

36 Tsou, K. and Jang, C.S., Studies on the site of analgesic ac- tion of morphine by intracerebral microinjection, Sci. Sin., 13 (1964) 1099-1109.

37 Urca, G. and Liebeskind, J.C., Electrophysiological indices of opiate action in awake and anesthetized rats, Brain Research, 161 (1979) 162-166.

38 Watkins, L.R. and Mayer, D.J., The organization of endogenous opiate and nonopiate pain control systems, Science, 216 (1982) 1185-1192.

39 Willis, W.D. In H. Autrum, D. Ottoson, E.R. Perl and R.F. Schmidt (Eds.), Progress in Sensory Physiology, Vol. 3, Springer, Berlin, 1982, pp. 1-158.

40 Worthington, E.L. Jr. and Martin, G.A., A laboratory analysis of response to pain after training in three Lamaze techniques, J. Psychosom. Res., 24 (1980) 109-116.

41 Yaksh, T.L. and Rudy, T.A., Narcotic analgetics: CNS sites and mechanisms of action as revealed by intracerebral injection techniques, Pain, 4 (1978) 299-359.

42 Zamir, N. and Segal, M., Hypertension-induced analgesia: changes in pain sensitivity in experimental hypertensive rats, Brain Research, 160 (1979) 170-173.

43 Zamir, N., Simantov, R. and Segal, M., Pain sensitivity and opioid activity in genetically and experimentally hypertensive rats, Brain Research, 184 (1980) 299-310.

44 Zorman, G., Hentall, I.D., Adams, J.E. and Fields, H.L., Naloxone-reversible analgesia produced by microstimulation in the rat medulla, Brain Research, 219 (1981) 137-148. 